\title{
Kommentar auf Anforderung der Schriftleitung
}

\author{
M. Imhof \\ Chirurgische Universitätsklinik, Würzburg
}

Die funktionserhaltende Therapie der Colitis ulcerosa und Adenomatosis coli ist jetzt mehr als eine Dekade alt. Gestützt auf die Erfahrungen der kontinenten Ileostomie nach Kock, entwickelte Parks einen S-förmigen Dünndarmbeutel zur sog. kontinenten ileoanalen Anastomose. Utsunomiya experimentierte ebenfalls Ende der 70er Jahre mit der kontinenten ileoanalen Anastomose, wobei er die Probleme des S-Pouch durch einfache Faltung des terminalen Ileums im Sinne eines sog. J-Pouch überwand. Diese Methode hat sich jetzt nach verschiedenen Modifikationen weltweit am häufigsten durchgesetzt. Trotz ihrer relativen technischen Einfachheit ist auch diese Methode jedoch durch eine nicht unbeträchtliche postoperative Morbidität belastet, wie lokale septische Prozesse, schwere Pouchitiden und schwer behandelbare funktionelle Probleme. Grund genug also, um trotz der weitgehenden Standardisierung der verschiedenen PouchVerfahren nach neuen Alternativen im Bereich der restaurativen Proktokolektomie zu suchen.

Generell lassen sich die postoperativen Folgezustände nach einer Proktokolektomie vorwiegend in 2 Problemkreise unterteilen:

- die ausgeprägte propulsive Dauerperistaltik des Dünndarms mit häufigen und unangenehmen Stuhlzwängen und

- die mangelnde Reservoirkapazität für die enzymreiche und aggressive Fäzes des Restdünndarms.

Die verschiedenen, bisher routinemäßig angewandten Pouch-Verfahren lösen diese komplexe Problematik durch die Eröffnung und gegenläufige Anordnung von Dünndarmschlingen mehr oder weniger befriedigend: Die orthograde propulsive Peristaltik wird durch die gegenläufige Anordnung von Dünndarmschlingen gebremst und die Reservoirkapazität erhöht.

In der vorliegenden Arbeit wird eine Methode vorgestellt, die von ihrer gedanklichen Konzeption her sehr

Korrespondenz an: Priv.-Doz. Dr. M. Imhof, Chirurgische Universitätsklinik Würzburg, Josef-Schneider-Straße 2, W-8700 Würzburg interessant ist: die Bildung eines intrapelvinen Dünndarmreservoirs ohne Eröffnung des Darmlumens und ohne gegenläufige Anordnung von Dünndarmschlingen. Dadurch entfallen die langen und komplikationsträchtigen Nahtreihen, die zeitlich aufwendige sind oder andererseits durch das Einbringen von Metallmaterial von Stapler-Anastomosen bei entzündlichen Darmerkrankungen nicht unproblematisch sind.

Die Methode leitet sich aus der plastisch-rekonstruktiven Chirurgie beim Verschluß größerer Hautdefekte her. Das Prinzip basiert auf der kontinuierlichen Expansion der Dünndarmwand durch einen intraluminal liegenden Gewebeexpander. Der Expander ist mit einem subkutan plazierten Ventil zur kontinuierlichen Ballonierung der Dünndarmwand konnektiert. Nach den vorliegenden tierexperimentellen Ergebnissen wird auf diese Weise ein Volumengewinn erzielt, der das 5 fache des Ausgangsvolumens des Dünndarms einnimmt. Außerdem resultiert aus der zunehmenden Expansion ein objektiver Gewebegewinn des Dünndarms, was durch histologische Serienuntersuchungen belegt werden konnte.

In der vorliegenden Versuchsreihe wurden allerdings nur der objektive Gewebegewinn durch histologische Untersuchungen und die Volumenzunahme des ballonierten Dünndarmsegments radiologisch ermittelt. Auf das entscheidende Kriterium jedes Pouch-Verfahrens, nämlich die Defäkationsfrequenz und die Kontinenzfunktion, wird in der vorliegenden Arbeit nur sehr kurz eingegangen: „... alle Versuchstiere waren kontinent, die Defäkationsfrequenz betrug 3,5 .... Von Interesse wäre in diesem Zusammenhang eine etwas genauere Charakterisierung der Schließmuskelfunktion nach Anschluß dieses Reservoirs an den verbliebenen Rektumzylinder gewesen.

Funktionelle, d.h. elektromanometrische Untersuchungen an dieser neuen Art des Rektumersatzes fehlen: Aus der histologisch nachgewiesenen objektiven $\mathrm{Zu}$ nahme an glatter Muskulatur müßte eigentlich eine Zunahme der propulsiven Peristaltik mit einer Erhöhung der Defäkationsfrequenz resultieren. Andererseits hat die chronische Überdehnung von glatter Muskulatur eine Paralyse der motorischen Aktivität zur Folge. Wie ent- 
leert sich also dieser Pouch gegen den Widerstand des Analsphinkters und wie verhält sich die elektrische und mechanische Aktivität in Abhängigkeit von verschiedenen Füllungszuständen dieses Dünndarm-Pouch?

Einen weiterer Dreh- und Angelpunkt der verschiedenen Pouch-Verfahren stellt die veränderte Compliance des Dünndarms dar. Um die einzelnen Verfahren hinsichtlich ihrer Funktionsweise vergleichen zu können, sind Meßdaten über das kapazitive Verhalten vor und nach der Pouch-Anlage wichtig.

Letztendlich bleibt die Frage offen, ob diese PouchVariante biodynamisch, architektonisch und funktionell eine neue Entität in Konkurrenz zu den herkömmlichen Pouch-Verfahren darstellt. Letztendlich wird zwar eine kontrollierte, jedoch immerhin eine chronische Ileussituation erzeugt. Exzellent ist allerdings die Defäkationsfrequenz von 3,5/Tag.

Zum Schluß sei noch auf die Problematik der Implantation von Fremdmaterial hingewiesen. In den vorliegenden tierexperimentellen Untersuchungen wurden septische Probleme im Bereich des subkutan verlagerten Kunststoffventils dokumentiert. Besonders bei entzündlichen Darmerkrankungen mit ihrer komplexen immunologischen Problematik ist die Verwendung von Fremdmaterial generell als kritisch einzustufen. Der diesbezügliche Hinweis der Autoren, daß beim Menschen der Expander vor der ileoanalen Anastomose plaziert werden könnte und das Ventil per vias naturales ausgeleitet werden könnte, erscheint ebenso nicht ganz unproblematisch: Bei der Aufdehnung des Gewebeexpanders ist mit einem Zug auf die eh schon kritische ileoanale Anastomose zu rechnen. Außerdem wären hochgradig unangenehme Sensationen im Beckenbereich wahrscheinlich.

Zusammenfassend stellt sich aus der Sicht des Kommentators das Problem folgendermaßen dar:

Das vorgestellte Prinzip ist von seiner Konzeption her sehr interessant: Ein Dünndarmbeutel wird ohne gegenläufig angeordnete Dünndarmschlingen und ohne lange Nahtreihen kreiert. Die Defäkationsfrequenz nach dieser Art von Dünndarmbeutel ist frappierend gut. Andererseits wird jedoch eine weitergehende, experimentelle Unterfütterung dieser Methodik vermißt: Es fehlen funktionelle, d.h. elektromanometrische Untersuchungen, die eine definitive Zuordnung und Vergleichbarkeit mit anderen Pouch-Verfahren zulassen. Die vorgestellte Konzeption sollte experimentell unbedingt weiterverfolgt werden. Nachteilig erscheint die Implantation eines Silikonexpanders gerade bei entzündlichen Darmerkrankungen.

Hinsichtlich des histologisch nachgewiesenen Gewebegewinns könnte diese Methode eine interessante Komponente beim Kurzdarmsyndrom darstellen. 